\title{
Detection of CS in Neptune's atmosphere from ALMA observations
}

\author{
R. Moreno ${ }^{1}$, E. Lellouch ${ }^{1}$, T. Cavalié ${ }^{1}$, and A. Moullet ${ }^{2}$ \\ ${ }^{1}$ LESIA-Observatoire de Paris, CNRS, Université Paris 06, Université Paris-Diderot, 5 place Jules Janssen, 92195 Meudon, France \\ e-mail: raphael.moreno@obspm.fr \\ 2 NRAO, 520 Edgemont Road, 22903 Charlottesville, VA, USA \\ Received 29 June 2017 / Accepted 15 September 2017
}

\section{ABSTRACT}

\begin{abstract}
Context. The large and vertically non-uniform abundance of CO in Neptune's atmosphere has been interpreted as the result of past cometary impact(s), either single or distributed in size and time, which could also be at the origin of Neptune's HCN.

Aims. We aim to provide observational support for this scenario by searching for other comet-induced species, in particular carbon sulfide (CS) which has been observed continuously in Jupiter since the 1994 Shoemaker-Levy 9 impacts.

Methods. In April 2016 we used the ALMA interferometer to search for CS(7-6) at $342.883 \mathrm{GHz}$ in Neptune.

Results. We report on the detection of CS in Neptune's atmosphere, the first unambiguous observation of a sulfur-bearing species in a giant planet beyond Jupiter. Carbon sulfide appears to be present only at submillibar levels, with a column density of (2.0$3.1) \times 10^{12} \mathrm{~cm}^{-2}$, and a typical mixing ratio of $(2-20) \times 10^{-11}$ that depends on its precise vertical location. The favoured origin of CS is deposition by a putative large comet impact several centuries ago, and the strong depletion of CS with respect to CO - compared to the Jupiter case - is likely due to the CS sticking to aerosols or clustering to form polymers in Neptune's lower stratosphere.

Conclusions. The CS detection, along with recent analyses of the $\mathrm{CO}$ profile, reinforces the presumption of a large comet impact into Neptune $\sim 1000$ yr ago, that delivered CO, CS, and HCN at the same time.
\end{abstract}

Key words. planets and satellites: individual: Neptune - techniques: spectroscopic - submillimeter: planetary systems

\section{Introduction}

External sources of oxygen are ubiquitous in the giant planets and Titan. This is demonstrated from the presence, in all five objects, of condensible species such as $\mathrm{H}_{2} \mathrm{O}$ and $\mathrm{CO}_{2}$ at stratospheric levels (Feuchtgruber et al. 1997; Lellouch et al. 2002; Orton et al. 2014), and from non-vertically uniform distributions of CO in all four giant planets (Bézard et al. 2002; Cavalié et al. 2009, 2014; Lellouch et al. 2005; Hesman et al. 2007; Luszcz-Cook \& de Pater 2013). The CO abundance in Neptune's stratosphere (about $1 \mathrm{ppm}$ ) is by far the largest of all the giant planets, and the external $\mathrm{CO}$ component implies a timeaveraged influx of oxygen material of the order of $10^{8} \mathrm{~cm}^{-2} \mathrm{~s}^{-1}$ (Lellouch et al. 2005; Luszcz-Cook \& de Pater 2013), at least 3 orders of magnitude larger than for Uranus. Building on the similarity with Jupiter, where the Shoemaker-Levy 9 (SL9) impacts injected massive amounts of fresh $\mathrm{CO}$ that added to the pre-existing levels on the planet, the preferred explanation for Neptune's CO is delivery from past cometary impacts in the form of a single large impact $\sim 200 \mathrm{yr}$ ago (Lellouch et al. 2005; Hesman et al. 2007) or of a distribution of impactors in size and time (Luszcz-Cook \& de Pater 2013). A comet impact would also supply HCN (Lellouch et al. 2005), although other sources of Neptune's HCN are possible (Marten et al. 1993; Lellouch et al. 1994).

The SL9 impacts also generated numerous sulfur species in Jupiter's stratosphere, including $\mathrm{S}_{2}, \mathrm{H}_{2} \mathrm{~S}, \mathrm{CS}, \mathrm{CS}_{2}$, and OCS (Noll et al. 1995; Lellouch et al. 1995). These species, except CS and $\mathrm{CS}_{2}$, have short atmospheric lifetimes (Moses 1996). In contrast, CS has been continuously observed to be present in Jupiter's atmosphere over the last $20 \mathrm{yr}$, with a relatively slow decay rate. This makes CS an attractive target in the framework of the comet impact scenario for Neptune. We report here the first detection of CS in Neptune's atmosphere, using ALMA.

\section{Observations and data reduction}

We observed Neptune with ALMA on three occasions between April 23 and 30, 2016. At that time the array included 39-41 $12 \mathrm{~m}$ antennas in configuration $\mathrm{C} 36-2 / 3$, providing a synthetic beam of about $0.6^{\prime \prime}$. The spectral set-up included the CS(7-6) line at $342.883 \mathrm{GHz}^{1}$ at a spectral resolution of $1 \mathrm{MHz}$. The $\mathrm{CO}(3-2)$ and $\mathrm{HCN}(4-3)$ lines were also covered. Observations (see details in Table 1) used the strong quasar J0006-0623 to calibrate the RF bandpass and the quasar J2246-1206 as phase calibrator. Initial data reduction was performed under the CASA software (i.e. the ALMA data reduction pipeline ${ }^{2}$ ).

The resulting calibrated visibilities were then exported into the GILDAS package ${ }^{3}$ for i) an improved amplitude calibration by scaling the three datasets to a single reference Neptune's angular diameter of $2.24^{\prime \prime}$; ii) a self-calibration technique using Neptune's continuum, which was also used as the absolute flux reference, yielding to an accuracy of 5\%; and iii) a spectral extraction by fitting the data directly in the UV-plane (i.e. the Fourier plane, as opposed to the image plane), providing the disk-averaged flux with optimum signal-to-noise ratio $(\mathrm{S} / \mathrm{N})$. These steps resulted in the detection of CS in three independent datasets (Fig. 1). The averaged line, which shows a contrast of $55 \mathrm{mJy}$ (i.e. $0.22 \%$ of Neptune's continuum) with a $\mathrm{S} / \mathrm{N}$ of 7 and a linewidth of $5.2 \mathrm{MHz}$ was used for modelling.

\section{Modelling and results}

Data were modelled with a standard radiative transfer model fully accounting for spherical geometry and broadening due to planetary rotation, developed for Jupiter (Moreno et al. 2001)

\footnotetext{
1 Two slightly different frequency setups were used on April 23-30.

2 https://casa.nrao.edu/

http://wWw.iram.fr/IRAMFR/GILDAS
} 
Table 1. ALMA observations of Neptune.

\begin{tabular}{cccccc}
\hline $\begin{array}{c}\text { UT start date } \\
\text { yyyy mm dd.ddd }\end{array}$ & $\begin{array}{c}\Delta^{1} \\
{[\mathrm{AU}]}\end{array}$ & $\begin{array}{c}\theta^{2} \\
{\left[{ }^{\prime \prime}\right]}\end{array}$ & $\mathrm{A}^{3}$ & $\begin{array}{c}T_{\text {int }}^{4} \\
(\mathrm{~min})\end{array}$ & $\begin{array}{c}\mathrm{PWV}^{5} \\
(\mathrm{~mm})\end{array}$ \\
\hline 20160423.477 & 30.559 & 2.234 & 41 & 20 & 0.9 \\
20160423.525 & 30.559 & 2.234 & 39 & 20 & 0.9 \\
20160430.484 & 30.462 & 2.241 & 41 & 20 & 1.4 \\
\hline
\end{tabular}

Notes. ${ }^{(1)}$ Geocentric distance. ${ }^{(2)}$ Apparent diameter at $p=1$ bar. ${ }^{(3)}$ Number of active antennas. ${ }^{(4)}$ On-source integration time. ${ }^{(5)}$ Precipitable water vapour.

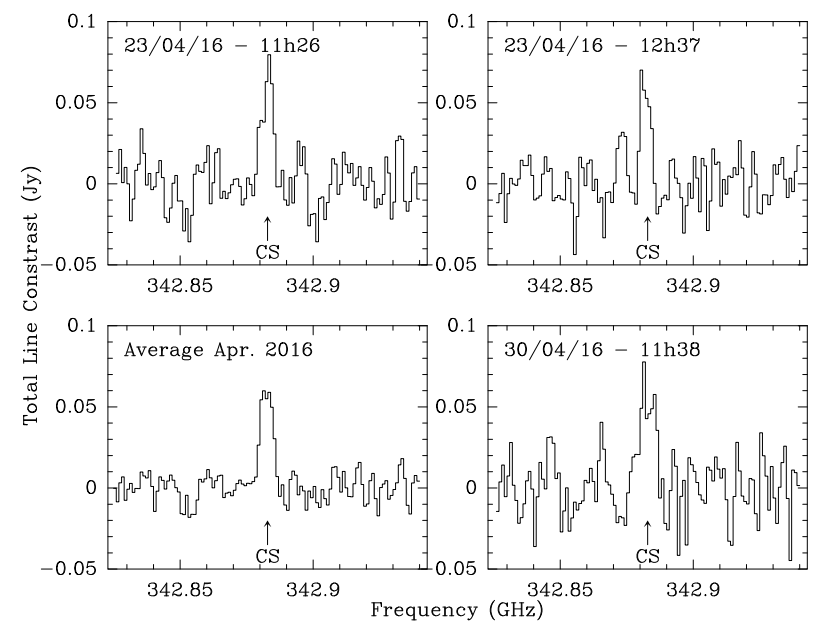

Fig. 1. ALMA individual observations of CS(7-6) at $342.8828503 \mathrm{GHz}$ on Neptune at different dates over April 23-30, 2016, and averaged spectrum (lower left panel). Both horizontal and vertical polarisations are averaged. Observations are scaled in intensity to the conditions of April 30, 2016. The spectral resolution is $\Delta v=1.0 \mathrm{MHz}$.

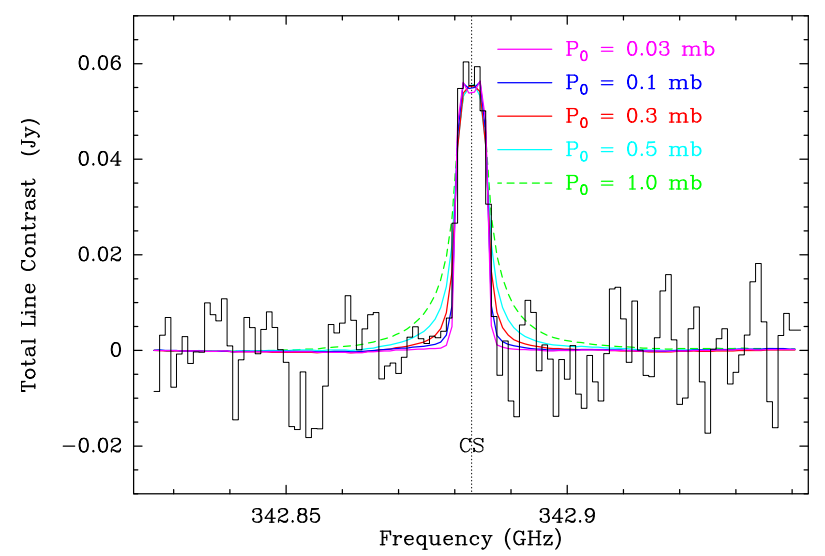

Fig. 2. Models of the CS(7-6) line, assuming a uniform mixing ratio distribution of CS at pressure levels lower than $\mathrm{P}_{0}$, where $\mathrm{P}_{0}$ is varied from 0.03 to 1.0 mbar (cases $A$ to $E$ in Table 2). These models are compared with the averaged spectrum shown in Fig. 1.

and also used for Neptune (Lellouch et al. 2005, 2015). Opacity sources of $\mathrm{CS}, \mathrm{CO}$, and $\mathrm{HCN}$ were included (using line parameters from Pickett et al. 1998), also included was the collision-induced absorption opacity due to the main compounds of Neptune's atmosphere, $\mathrm{H}_{2}-\mathrm{H}_{2}, \mathrm{H}_{2}-\mathrm{He}$, and $\mathrm{H}_{2}-\mathrm{CH}_{4}$, using codes developed by Borysow et al. (1985, 1988), Borysow \& Frommhold (1986). We adopted a He mole fraction of 0.149 (Burgdorf et al. 2003) and the $\mathrm{CH}_{4}$ vertical distribution of Lellouch et al. (2015). The Neptune thermal profile, taken from Lellouch et al. (2010), is shown in Fig. 3, along

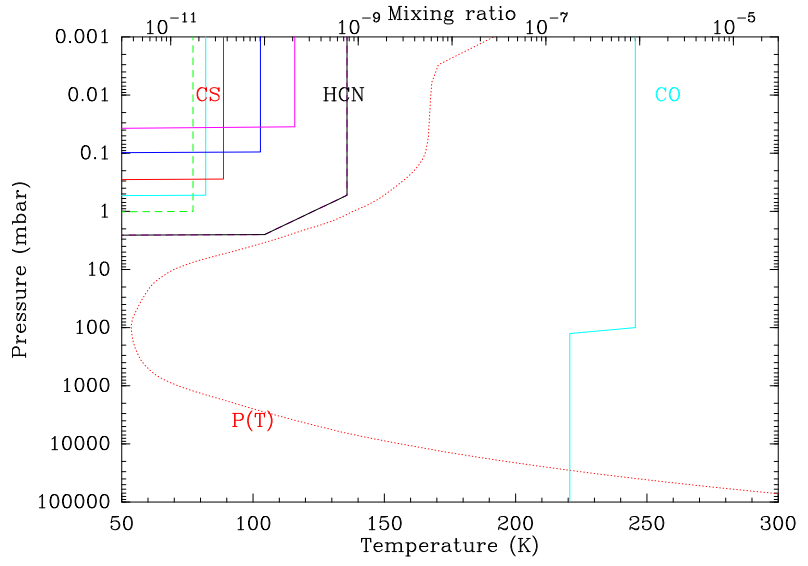

Fig. 3. Neptune atmospheric model: thermal profile and vertical distributions of $\mathrm{CO}, \mathrm{HCN}$, and $\mathrm{CS}$.

Table 2. Retrieved CS mixing ratio and column density assuming uniform profiles at pressure less than $\mathrm{P}_{0}$.

\begin{tabular}{cccc}
\hline \hline Profile & $\mathrm{P}_{0}(\mathrm{mbar})$ & Mixing ratio & Column $\left(\mathrm{cm}^{-2}\right)$ \\
\hline $\mathrm{A}$ & 0.03 & $21.0_{-3.00}^{+3.00} \times 10^{-11}$ & $2.0 \times 10^{12}$ \\
$\mathrm{~B}$ & 0.1 & $9.05_{-1.35}^{+1.45} \times 10^{-11}$ & $2.2 \times 10^{12}$ \\
$\mathrm{C}$ & 0.3 & $3.65_{-0.50}^{+0.55} \times 10^{-11}$ & $2.5 \times 10^{12}$ \\
$\mathrm{D}$ & 0.5 & $2.36_{-0.36}^{+0.36} \times 10^{-11}$ & $3.1 \times 10^{12}$ \\
\hline $\mathrm{E}^{a}$ & 1.0 & $1.73_{-0.25}^{+0.25} \times 10^{-11}$ & $4.3 \times 10^{12}$ \\
\hline
\end{tabular}

Notes. Mixing ratios include uncertainties in ALMA calibration and statistical uncertainties. ${ }^{(a)}$ Modelled linewidth is too large.

with optimised $\mathrm{CO}$ and $\mathrm{HCN}$ profiles, retrieved from the high $\mathrm{S} / \mathrm{N}$ observations of $\mathrm{CO}$ and $\mathrm{HCN}$ (not shown). As the CS pressure broadening coefficient $(\gamma)$ was not found in the literature, we nominally used the value of the $\operatorname{HCN}(4-3)$ line, $\gamma=0.121 \mathrm{~cm}^{-1} / \mathrm{atm}$ with a temperature exponent $n=0.74$ (Landrain et al. 1997; Rohart et al. 2007).

The observed CS linewidth is consistent with broadening essentially due to the planet rotation, with at most minor Lorenz broadening. This precludes a measurement of the CS vertical profile, except for an upper limit on the pressure level at which it resides. Testing uniform CS profiles above a pressure level $\mathrm{P}_{0}$, we inferred that the maximum allowable $\mathrm{P}_{0}$ is $\sim 0.5$ mbar (Figs. 2 and 3). As the CS line is fully optically thin (e.g. $\tau=0.047$ for $\mathrm{P}_{0}=0.3$ mbar), the best determined quantity is the CS column density found to be in the range $(2.0-3.1) \times 10^{12} \mathrm{~cm}^{-2}$, while the CS mixing ratio above $\mathrm{P}_{0}$ essentially scales as $1 / \mathrm{P}_{0}$ and is $\sim 9 \times 10^{-11}$ for $\mathrm{P}_{0}=0.1 \mathrm{mbar}$. Thus, CS is restricted to the upper stratosphere, similar to (and even higher than) $\mathrm{HCN}$, and in sharp contrast with CO. We also tested a case with $\gamma=0.067 \mathrm{~cm}^{-1} / \mathrm{atm}$ and $n=0.64$ (i.e. the $\mathrm{CO}(3-2)$ broadening parameters, Sung 2004; Mantz et al. 2005). In this case the CS mixing ratio and column density is about $10 \%$ lower, and the maximum allowed $\mathrm{P}_{0}$ is $\sim 0.9$ mbar. We thus conservatively conclude that the bulk of CS resides at pressures of less than 1 mbar.

\section{Discussion}

Our detection of CS in Neptune is the first unambiguous observation of a sulfur-bearing compound in a giant planet beyond Jupiter. Although $\mathrm{H}_{2} \mathrm{~S}$, which has been established in Jupiter (Niemann et al. 1996), has been tentatively reported as 
well in the deep atmospheres of Saturn, Uranus, and Neptune (Briggs \& Sackett 1989; de Pater et al. 1991), this inference was based on the microwave spectrum in which the contributions of $\mathrm{H}_{2} \mathrm{~S}$ and $\mathrm{NH}_{3}$ are difficult to disentangle. At any rate, the putative presence of $\mathrm{H}_{2} \mathrm{~S}$ in Neptune is almost certainly unrelated to that of CS. Indeed, any tropospheric $\mathrm{H}_{2} \mathrm{~S}$ is expected to condense out near the 10 bar level, and therefore cannot serve as a source for CS at submillibar levels.

Prior to our measurements, and with the same motivation to confirm the comet impact scenario for CO, Iino et al. (2014) used the ASTE $10 \mathrm{~m}$ telescope to target a suite of sulfur-bearing species ( $\mathrm{CS}, \mathrm{OCS}, \mathrm{H}_{2} \mathrm{CS}, \mathrm{H}_{2} \mathrm{~S}, \mathrm{SO}, \mathrm{C}_{3} \mathrm{~S}$, and $\mathrm{SO}_{2}$ ). None of these species was detected, but stringent upper limits were obtained. For CS, assuming uniform mixing at $p<6$ mbar, Iino et al. (2014) obtained a CS mixing ratio upper limit of $1.8 \times 10^{-10}$. Using for reference the $\mathrm{CO}$ mixing ratio measured by Hesman et al. (2007) for this pressure range (i.e. $2.2 \times 10^{-6}$ ), this implied a CS/CO upper limit of $8 \times 10^{-5}$.

As detailed above, the CS stratospheric mixing ratio is $2.4 \times$ $10^{-11}$ assuming that $\mathrm{CS}$ is present only at pressures lower than 0.5 mbar, and $\sim 4$ times higher if CS is restricted to $p<0.1 \mathrm{mbar}$. The most recent analyses of CO line profiles (combining data from Herschel/SPIRE and ground-based observations, Moreno et al., in prep.) indicate a CO tropospheric (resp. stratospheric) mixing ratio of $0.2 \times 10^{-6}$ (resp. $1.0 \times 10^{-6}$ ), indicative of a stratospheric excess of $0.8 \times 10^{-6}$ of external origin that extends all the way down to 100 mbar. The above values for CS imply a $\mathrm{CS} / \mathrm{CO}$ volume ratio of $(3-11) \times 10^{-5}$ in the region where both species are present. However, as the external CO component extends much deeper than CS, a more relevant figure can be obtained by using the ratio of the column densities (or total masses) instead of the volume mixing ratios. The "external CO" column density $\left(0.8 \times 10^{-6}\right.$ mixing ratio above $\left.100 \mathrm{mbar}\right)$ is approximately $1.8 \times 10^{19} \mathrm{~cm}^{-2}$. This gives a CS/CO column ratio of $1.4 \times 10^{-7}$.

As already noted by Iino et al. (2014), Neptune's stratospheric CS/CO ratio is much lower than Jupiter's. Based on data taken within 1 day after an impact, Lellouch et al. (1995) and Lellouch (1996) determined $\mathrm{CS} / \mathrm{CO}=3 \times 10^{-3}$ by mass. The CS/CO ratio was found to be higher $(\sim 0.01$ by mass) $\sim 1$ week after the impacts (Moreno et al. 2001) and continued to increase to reach $\sim 0.036$ by mass in the $1995-1998$ time frame (Moreno et al. 2003). This evolution pointed to a likely photochemical build-up of CS from $\mathrm{S}_{2}$ (Moses 1996), which was heavily produced from the impacts (Noll et al. 1995). The latter (i.e. long-term) value for $\mathrm{CS} / \mathrm{CO}$ is probably more relevant for a comparison with Neptune. Thus, it appears that the CS/CO in Neptune's submillibar region is at least 200-1000 times smaller than in Jupiter's, and even much lower $\left(\sim 1.6 \times 10^{5}\right.$ times $)$ if Neptune's CS/CO ratio is considered in terms of column density.

At face value this might be taken against the comet-impact source scenario for Neptune's CS (and CO and HCN). However, based on an unpublished monitoring of $\mathrm{CO}, \mathrm{CS}$, and $\mathrm{HCN}$ in Jupiter over 1996-2006, Moreno \& Marten (2006) found that during that period, the total $\mathrm{CO}, \mathrm{CS}$, and $\mathrm{HCN}$ masses underwent global decreases by factors of $\sim 6, \sim 12$, and $\sim 8$, respectively. For CS, a similar mass decrease of a factor of 11 was reported by Iino et al. (2016), but over 1996-2013. While the uncertainties on these figures are too large (typically a factor 2) to confidently conclude, they leave open the possibility that the evolution timescales for the different species are different. For example, adopting these nominal loss factors for $\mathrm{CO}$, $\mathrm{CS}$, and $\mathrm{HCN}$ over a ten-year interval leads to e-folding decay timescales of 5.4, 4.0, and $4.6 \mathrm{yr}$ for $\mathrm{CO}, \mathrm{CS}$, and $\mathrm{HCN}$. The assumption of exponential decay is not proven, but reasonable for a unimolecular loss reaction such as sticking or a bimolecular one in which the other reactant is much more abundant. Although the direct applicability to Neptune is obviously hazardous to say the least, extrapolating this over a 200-yr timespan would lead to a depletion which is $\sim 3 \times 10^{5}$ times stronger for $\mathrm{CS}$ than for $\mathrm{CO}$. This effect is comparable to what is required to account for the much lower CS/CO mass ratio for Neptune (by a factor of $\sim 1.6 \times 10^{5}$ ) compared to Jupiter. We also note that the same extrapolation over $200 \mathrm{yr}$ would lead to a depletion of $\mathrm{CS} / \mathrm{HCN}$ by 520 , qualitatively matching the higher HCN/CS mass ratio on Neptune compared to Jupiter (by a factor of $\sim 40$, based on Fig. 3).

The other striking difference between Jupiter and Neptune is in the relative vertical distributions of CS and CO. In Jupiter, the two-decade monitoring indicates that the three SL9 related species, $\mathrm{CO}, \mathrm{CS}$, and $\mathrm{HCN}$, have diffused downward from an initial deposition level of 0.1 mbar (Lellouch et al. 1995) to 0.3 mbar between 1998-2006 (Moreno et al. 2003; Moreno \& Marten 2006) and appear to be roughly vertically colocated. In Neptune, CS is exclusively present in the submillibar level, while external CO invades the lower stratosphere, down to 6 mbar, 20 mbar, 2.5-100 mbar, and 100 mbar according respectively to Hesman et al. (2007), Lellouch et al. (2005), Luszcz-Cook \& de Pater (2013, Fig. 19), and Moreno et al. (in prep.). Therefore, if CS was injected/produced following the same comet impact that is invoked for $\mathrm{CO}$, it has not followed the same time evolution on the long term. The same is true for Neptune's HCN which -even if deposited by an ancient impactis currently present at pressures lower than $\sim 3$ mbar only, due to condensation. Carbon sulfide is stable (e.g. against photolysis) in the gas phase, but is a highly reactive species that polymerises on walls during laboratory experiments (Moltzen et al. 1988) and therefore has no measurable vapour pressure. Thus, while it does not "condense" in the traditional sense, CS can probably easily stick to aerosol surfaces or otherwise cluster together to form CS polymers; heterogeneous reactions on grain surfaces may also convert some of the $\mathrm{CS}$ to $\mathrm{H}_{2} \mathrm{CS}$ or to $\mathrm{CH}_{3} \mathrm{CS}$ via hydrogenation (J.-C. Loison, priv. comm.). Neptune's hazes extend up to the mbar level, fed by the condensation of species such as HCN, $\mathrm{H}_{2} \mathrm{O}, \mathrm{C}_{4} \mathrm{H}_{2}$, and other heavier hydrocarbons. In the framework of the "old impact" hypothesis, this could be the reason for the vastly different vertical distributions of $\mathrm{CO}, \mathrm{HCN}$, and $\mathrm{CS}$ in Neptune. In Jupiter, the three species are still co-located given their recent deposition, but may vertically segregate in the longer term due to the same processes.

The comet impact hypothesis of Lellouch et al. (2005) seems to be a viable explanation for $\mathrm{CO}, \mathrm{HCN}$, and $\mathrm{CS}$ in Neptune. Lellouch et al. (2005) estimated a 200-yr impact age by equating it to the timescale $\left(\tau=2 \mathrm{H}^{2} / \mathrm{K}\right)$ required for diffusion down to the $20 \mathrm{mbar}$ level, using an eddy $\mathrm{K}$ of $3000 \mathrm{~cm}^{2} \mathrm{~s}^{-1}$ at $20 \mathrm{mbar}$ and an atmospheric scale height $H=30 \mathrm{~km}$, and inferred the diameter of the impactor to be $D=2 \mathrm{~km}$ based on the amount of external $\mathrm{CO}$ they determined. In a slightly different formulation and considering lower values for K (e.g. 200-2000 $\mathrm{cm}^{2} \mathrm{~s}^{-1}$ at the tropopause), Luszcz-Cook \& de Pater (2013) inferred timescales of 100-1000 yr. Furthermore, the most recent determinations of the CO profile (Luszcz-Cook \& de Pater 2013; Moreno et al., in prep.) indicate a $0.8 \times 10^{-6} \mathrm{CO}$ stratospheric excess down to the $\sim 100$ mbar level. As this represents 8 times more external $\mathrm{CO}$ than reported by Lellouch et al. (2005) (a $0.5 \times 10^{-6}$ excess down to 20 mbar), we conclude that the single-impact scenario might have to be revised in favour of a slightly older - up to $1000 \mathrm{yr}$ ago - but even bigger $(D=4 \mathrm{~km})$ comet impact. 
Following the approach used by Bézard et al. (2002) to determine the origin of the non-SL9 CO stratospheric component on Jupiter, Luszcz-Cook \& de Pater (2013) found that a permanent influx of small (sub-km size) comets is another possibility for Neptune's CO, perhaps preferable to the single, large-comet explanation given the statistical rarity of large impacts. We note, however, that the $\mathrm{CO}$ equivalent flux into Neptune is likely to be in excess of $10^{8} \mathrm{~cm}^{2} \mathrm{~s}^{-1}\left(8 \times 10^{8} \mathrm{~cm}^{2} \mathrm{~s}^{-1}\right.$ from scaling the Lellouch et al. (2005) flux by 8 , and $(0.5-20) \times 10^{8} \mathrm{~cm}^{2} \mathrm{~s}^{-1}$ according to the Luszcz-Cook \& de Pater (2013) physical models), while the non-SL9 stratospheric CO on Jupiter requires a time-averaged flux of only $(1.5-10) \times 10^{6} \mathrm{~cm}^{2} \mathrm{~s}^{-1}$ (Bézard et al. 2002). Cometary impacts have also been advocated as being responsible for the observed stratospheric CO in Saturn (Cavalié et al. 2010) and Uranus (Cavalié et al. 2014). While these two papers have considered the comet impact hypothesis in its "single large" impact version, a distribution of small comets is probably a viable solution for these two planets as well. But the very large external $\mathrm{CO}$ mixing ratio $\left(0.8 \times 10^{-6}\right)$ for Neptune, compared to $(3-10) \times 10^{-9}$ for Jupiter (Bézard et al. 2002), $(1-3) \times 10^{-8}$ for Saturn (Cavalié et al. 2010), and (0.7-1) $\times 10^{-8}$ for Uranus (Cavalié et al. 2014), combined with the fact that statistical ecliptic comet impact rates (Levison et al. 2000) are not particularly enhanced on Neptune compared to other giant planets (e.g. no more than a factor of 2 more frequent than on Uranus), tends to argue for a unique $\sim 4 \mathrm{~km}$ comet impact on Neptune. Such an event may seem unlikely, but based on the Zahnle et al. (2003) cratering rates, we estimated that it occurs every 3400-4100 yr.

Alternate hypotheses do not seem easy to imagine. Lellouch et al. (1994) proposed that Triton's escaping atmosphere could be a source for the nitrogen present in Neptune's $\mathrm{HCN}$ and found this mechanism to require a total nitrogen infall rate of $5 \times 10^{24} \mathrm{~N}$ atoms s $\mathrm{s}^{-1}$, in plausible agreement with estimates of Triton's escape rate, and of ionisation and transport rates of nitrogen to Neptune. However, the process is likely to be irrelevant for CS, given the lack of any detected atmospheric or surface sulfur-bearing species on Triton (see e.g. Grundy et al. 2010, for a high S/N near-IR spectrum of Triton).

Given the elemental abundance composition of cometary dust (Jessberger \& Kissel 1991, i.e. O/S = 12.4), interplanetary dust particles also represent a source of oxygen and other volatiles, with an $\mathrm{O} / \mathrm{S}$ ratio broadly consistent with solar composition (chondritic $\mathrm{O} / \mathrm{S}=8.5$, photospheric solar $\mathrm{O} / \mathrm{S}=31.7$; Lodders 2003). Thus, in terms of chemistry, interplanetary dust particles (IDP) may be equally viable sources of sulfur as cometary impacts. However, the oxygen influx required to explain the abundances of $\mathrm{CO}$ on Neptune is vastly different $\left(\gtrsim 10^{8} \mathrm{~cm}^{2} \mathrm{~s}^{-1}\right.$, Lellouch et al. 2005; Luszcz-Cook \& de Pater 2013; Hesman et al. 2007) from that needed to account for the $\mathrm{CO}, \mathrm{H}_{2} \mathrm{O}$, and $\mathrm{CO}_{2}\left(\sim(2-3) \times 10^{5} \mathrm{~cm}^{2} \mathrm{~s}^{-1}\right.$ Cavalié et al. 2014; Orton et al. 2014) on Uranus; instead, based on a dynamical interplanetary dust model, Poppe (2016) calculated an IDP oxygen flux only $\sim 5$ times larger than into the atmosphere of Uranus. In fact, Poppe (2016) and Moses \& Poppe (2017) find that a flux of IDPs is sufficient to account for the abundances of all three oxygen-bearing species on Uranus, as well as $\mathrm{H}_{2} \mathrm{O}$ on Neptune (and $\mathrm{CO}_{2}$, if $\mathrm{CO}$ is prescribed), but fails to explain Neptune's CO abundance. Based on their calculation of dust ablation, Moses \& Poppe (2017) conclude that the bulk of the stratospheric oxygen on Neptune originates from a cometary impact and not from IDPs.

In summary, the CS detection, along with recent analyses of the $\mathrm{CO}$ profile, reinforces the presumption of a large comet impact into Neptune up to $\sim 1000$ yr ago that delivered CO, CS, and $\mathrm{HCN}$ at the same time. The three species may have been produced in proportions that were initially similar to the SL9 events, but relative amounts were progressively altered by the preferential condensation/sticking of HCN near 3 mbar, and CS polymerisation or other chemical loss at submillibar levels.

Acknowledgements. This paper makes use of the following ALMA data: ADS/JAO.ALMA\#2015.1.01471.S (PI: R. Moreno). ALMA is a partnership of ESO (representing its member states), NSF (USA), and NINS (Japan), together with NRC (Canada), NSC and ASIAA (Taiwan), and KASI (Republic of Korea), in cooperation with the Republic of Chile. The Joint ALMA Observatory is operated by ESO, AUI/NRAO, and NAOJ. We thank J.-C. Loison and J.-C. Guillemin for useful discussions and J.I. Moses for her stimulating review. R. Moreno, E. Lellouch, and T. Cavalié wish to thank the Programme National de Planétologie of CNRS/INSU for funding.

\section{References}

Bézard, B., Lellouch, E., Strobel, D., Maillard, J.-P., \& Drossart, P. 2002, Icarus, 159,95

Borysow, A., \& Frommhold, L. 1986, ApJ, 304, 849

Borysow, J., Trafton, L., Frommhold, L., \& Birnbaum, G. 1985, ApJ, 296, 644 Borysow, J., Frommhold, L., \& Birnbaum, G. 1988, ApJ, 326, 509 Briggs, F. H., \& Sackett, P. D. 1989, Icarus, 80, 77

Burgdorf, M., Orton, G. S., Davis, G. R., et al. 2003, Icarus, 164, 244 Cavalié, T., Billebaud, F., Dobrijevic, M., et al. 2009, Icarus, 203, 531 Cavalié, T., Hartogh, P., Billebaud, F., et al. 2010, A\&A, 510, A88 Cavalié, T., Moreno, R., Lellouch, E., et al. 2014, A\&A, 562, A33 de Pater, I., Romani, P. N., \& Atreya, S. K. 1991, Icarus, 91, 220 Feuchtgruber, H., Lellouch, E., de Graauw, T., et al. 1997, Nature, 389, 159 Grundy, W. M., Young, L. A., Stansberry, J. A., et al. 2010, Icarus, 205, 594 Hesman, B. E., Davis, G. R., Matthews, H. E., \& Orton, G. S. 2007, Icarus, 186, 342

Iino, T., Mizuno, A., Nakajima, T., et al. 2014, Planet. Space Sci., 104, 211 Iino, T., Ohyama, H., Hirahara, Y., Takahashi, T., \& Tsukagoshi, T. 2016, AJ, 152,179

Jessberger, E. K., \& Kissel, J. 1991, in Comets in the post-Halley era, eds. R. L. Newburn, Jr., M. Neugebauer, \& J. Rahe, Astrophys. Space Sci. Lib., 167, IAU Colloq. 116, 1075

Landrain, V., Blanquet, G., Lepère, M., Walrand, J., \& Bouanich, J.-P. 1997, J. Mol. Spectr., 182, 184

Lellouch, E. 1996, in The Collision of Comet Shoemaker-Levy 9 and Jupiter, eds. K. S. Noll, H. A. Weaver, \& P. D. Feldman, IAU Colloq., 156, 213

Lellouch, E., Romani, P. N., \& Rosenqvist, J. 1994, Icarus, 108, 112 Lellouch, E., Paubert, G., Moreno, R., et al. 1995, Nature, 373, 592 Lellouch, E., Bézard, B., Moses, J. I., et al. 2002, Icarus, 159, 112 Lellouch, E., Moreno, R., \& Paubert, G. 2005, A\&A, 430, L37 Lellouch, E., Hartogh, P., Feuchtgruber, H., et al. 2010, A\&A, 518, L152 Lellouch, E., Moreno, R., Orton, G. S., et al. 2015, A\&A, 579, A121

Levison, H. F., Duncan, M. J., Zahnle, K., Holman, M., \& Dones, L. 2000, Icarus, 143,415

Lodders, K. 2003, ApJ, 591, 1220

Luszcz-Cook, S. H., \& de Pater, I. 2013, Icarus, 222, 379

Mantz, A. W., Malathy Devi, V., Chris Benner, D., et al. 2005, J. Mol. Struct., 742, 99

Marten, A., Gautier, D., Owen, T., et al. 1993, ApJ, 406, 285

Moltzen, E. K., Klabunde, K. J., \& Senning, A. 1988, Chem. Rev., 88, 391

Moreno, R., \& Marten, A. 2006, BAAS, 38, 497

Moreno, R., Marten, A., Biraud, Y., et al. 2001, Planet. Space Sci., 49, 473

Moreno, R., Marten, A., Matthews, H. E., \& Biraud, Y. 2003, Planet. Space Sci., 51,591

Moses, J. I. 1996, in The Collision of Comet Shoemaker-Levy 9 and Jupiter, eds.

K. S. Noll, H. A. Weaver, \& P. D. Feldman, IAU Colloq., 156, 243

Moses, J. I., \& Poppe, A. R. 2017, Icarus, 297, 33

Niemann, H. B., Atreya, S. K., Carignan, G. R., et al. 1996, Science, 272, 846

Noll, K. S., McGrath, M. A., Trafton, L. M., et al. 1995, Science, 267, 1307

Orton, G. S., Moses, J. I., Fletcher, L. N., et al. 2014, Icarus, 243, 471

Pickett, H. M., Poynter, R. L., Cohen, E. A., et al. 1998,

J. Quant. Spectr. Rad. Transf., 60, 883

Poppe, A. R. 2016, Icarus, 264, 369

Rohart, F., Nguyen, L., Buldyreva, J., Colmont, J.-M., \& Wlodarczak, G. 2007, J. Mol. Spectr., 246, 213

Sung, K. 2004, J. Quant. Spectr. Rad. Transf., 83, 445

Zahnle, K., Schenk, P., Levison, H., \& Dones, L. 2003, Icarus, 163, 263 